\title{
Case report on hydatid cyst of the liver progressed to the lungs
}

\author{
Sree Keerthi Nethi*, Vyshnavi Biradavolu, Durga Prasad T. S.
}

Department of Pharmacy practice, Sri Padmavathi School of Pharmacy, Tiruchanoor, Tirupathi, Andhra Pradesh, India

Received: 24 January 2017

Accepted: 27 February 2017

\section{*Correspondence to:}

N. Sree Keerthi,

Email:

sreekeerthi.nethi@gmail.com

Copyright: (C) the author(s), publisher and licensee Medip Academy. This is an openaccess article distributed under the terms of the Creative Commons Attribution NonCommercial License, which permits unrestricted noncommercial use, distribution, and reproduction in any medium, provided the original work is properly cited

\begin{abstract}
Hydatid liver disease is commonly found in sheep farming areas. Hydatid cysts are confined to the liver and lungs in common. Many reports on hydatid cysts of liver and lungs were reported individually. Here, we report a case of hydatid cyst of the liver leading to the development of cysts in the lungs. The patient had previously underwent surgery for the removal of hydatid cysts in the liver but chemotherapy with albendazole was neither initiated immediately nor continued for 3 to 6 weeks after initiation. Later she presented with shortness of breath and cough along with fever and chills, for which she was diagnosed to have pleural effusion confirmed with pleural fluid analysis showing increase in ADA (Adenosine deaminase) and protein levels and USG (Ultrasonography) demonstrating loculations. Inspite of specific therapy, pleural effusion was not relieved and she was advised for CT chest which revealed multiple cysts in the lung. Suspecting for the liver cysts, USG abdomen was advised and it confirmed the presence of hydatid cysts in the liver. This helped to draw a conclusion that failure of earlier surgical intervention i.e excision of hydatid cyst of the liver lead to the development of cyst in the lungs that presented as pleural effusion. This case highlights the importance of chemotherapy along with surgical intervention before and after the surgery.
\end{abstract}

Keywords: Albendazole, Excision of cyst, Hydatid cyst, Liver, Lung

\section{INTRODUCTION}

Human echinococcosis or hydatidosis is a dog borne zoonotic infection, caused when the humans meet with accidental ingestion of eggs with larval stage of the dwarf tapeworm of the genus Echinococcus. ${ }^{-4}$ It is most commonly caused by the species Echinococcus granulosus and Echinococcus multilocularis that vary in geographic distribution. ${ }^{2}$ It's of major public health importance especially in sheep farming areas like Central Europe, Africa, South America, Newzeland, Australia, Central Asia and china because sheep is the intermediate host ${ }^{(4)}$. It is also evident from previous reports that in INDIA, Kurnool, Kadapa and Chittoor districts were more epidemic from Andhra Pradesh. ${ }^{5}$

Hydatid cyst usually affects the liver (50-70\%), followed by lungs (15-30\%) and rarely in other organs like spleen, kidney, bones and brain. ${ }^{1,7}$ Clinical manifestations vary based on the organ affected and the load and size of cyst affecting the organ. Usually, patient may remain asymptomatic for many years because cyst grows only 1$3 \mathrm{~cm}$ per year, due to which many cases may go undiagnosed. ${ }^{1}$ Hence this is highly fatal, for which immediate diagnostic and therapeutic intervention should be made. ${ }^{5}$

Clinical manifestations of hydatid echinococcosis vary based on the size and number of cysts. However, symptoms including fever, chills, nausea, dyspnea, dysphagia, pain in the right upper quadrant can be observed due to some cysts. Complications associated with the free rupture of the echinococcal cyst may cause anaphylaxis and smaller cysts may be released that can circulate to and lodge in other organs. ${ }^{4}$

The diagnosis of echinococcosis is mainly based on patient's history, clinical findings, hematological and serum biochemical profiles including serum Bilurubin, ALP, leucocytes, esinophils, serum protein. ${ }^{2}$ The 
confirmation can be made with the help of immunoelectrophoresis that demonstrate antibodies to antigen 5, whereas ELISA (Enzyme Linked Immuno Sorbent Assay) and indirect hemaggutination test are of initial choices that have $90 \%$ sensitivity. It is also evident that USG (Ultrasonography) helps in diagnosis, treatment and follow up of the patients, whereas CT scan can be preferred in cases where USG fails due to patient related difficulties such as obesity, excessive intestinal gas, abdominal wall deformities and previous surgery. ${ }^{3}$

Radical surgical procedure remains the main stay of therapy, whereas the conservative surgical procedure ends up with risk of developing anaphylactic reactions, chemical cholangitis and even relapses up to $20 \%$. This is because conservative procedure aims at sterilization and partial removal of cyst, whereas radical procedure aims at complete resection of the cyst. Surgery combined with chemotherapy before and after 8 weeks of surgery was found to be successful existing therapy. ${ }^{3}$ In case of hydatidosis of brain and heart, Percutaneous-AspirationInjection-Reaspiration (PAIR) drainage is considered lifesaving. ${ }^{1-3}$

\section{CASE REPORT}

A 28 years old female patient who was apparently normal 10 days before the admission, presented with chief complaints of breathlessness, which is increased on coughing, along with high grade, intermittent fever and mucoid and no productive cough since 7 days. She also had history of weight loss and loss of appetite (anorexia), but AFB (Acid Fast Bacilli) staining was negative. By occupation, she is a coolie besides which their family have goat farming that can be considered as the major risk factor for the development of this illness.

Her previous history revealed that she had undergone Excision of Hydatid cyst of the liver on $3^{\text {rd }}$ October 2016 and was advised for the review. Later on $1^{\text {st }}$ December 2016, she was prescribed with T.LARIGO (Chloroquine), T.REZQ (Quinine sulphate), T.ALBENO (Albendazole), T.AUGMENTIN (Amoxicillin + Clavulanic acid), and T.RANTAC (Ranitidine) for fever with chills, but her complaints were not resolved. In addition to these, she developed cough and shortness of breath, for which she was readmitted in Aasha hospital where she was diagnosed to have right pleural effusion with moderate to free fluid, and was advised with the therapeutic regimen including AUGMENTIN (Amoxicillin + Clavulanic acid), DOLO (Acetaminophen), DOXOVENT (Doxofylline), MULTIVITAMIN and REZQ (Quinine sulphate).

Between $4^{\text {th }}$ and $7^{\text {th }}$ November 2016, her laboratory profile picturizes that there was increase in WBC, Lymphocytes in blood and proteins and ADA (Adenosine deaminase) levels in pleural fluid and cytology and AFB staining were negative. ADA $63 \mathrm{U} / \mathrm{L}(>60)$ and proteins
$5.5 \mathrm{~g} / \mathrm{dl}(>3 \mathrm{~g} / \mathrm{dl})$ helped to draw the conclusion that this is an exudative type of pleural effusion.

Later on $10^{\text {th }}$ December 2016, she was presented with chief complaints of breathlessness, which is increased on coughing, along with high grade, intermittent fever and mucoid and no productive cough since 7 days. On the day of admission, ADA levels and proteins of pleural fluid were high in concentrations. With support from the laboratory investigations like USG chest which revealed loculated type of effusion with septations in the right cavity and USG abdomen that revealed Hydatid cyst of size $79 \times 73 \mathrm{~mm}$, it is evident that cyst excision was not successful in complete elimination of the cyst in liver, so that it was regenerated in the liver. Adding to this, CT chest revealed that there are multiple Hydatid cysts with internal loculations in right lobe and a cyst with calcified wall in the left lobe, from which conclusion can be drawn that cyst migration through the portal system into the lungs had lead to the development of Hydatid lung disease that presented as a clinical manifestation of right pleural effusion.

On the day of admission, patient was prescribed with Paracetamol, B. Complex Ranitidine, followed by the addition of Augmentin and diagnostic Thoracentesis that helped to rule out TB and later ALBENDAZOLE 450mg, was added to the regimen after confirmation of multiple Hydatid cysts from the USG report. Diclofenac was added from $14^{\text {th }}$ December 2016 to provide relief from abdominal pain. She was advised to continue same therapy till $24^{\text {th }}$ December 2016. From $12^{\text {th }}$ December 2106, Albendazole was prescribed with a 2 month cycle of alternating 4 weeks administration and 2 weeks pause and was advised to attend for the review.

\section{DISCUSSION}

Hydatidosis is a zoonotic, pathologic and parasitic infection that is caused by the ingestion of eggs of the tapeworm of the genus Echinococcus from the feces of infected dog, where dogs are the definitive host and the sheep is the secondary host. ${ }^{1}$ This case is consistent with previous studies in risk factor because the patient's family had sheep farming and also dogs are roaming near the house and she is from Kadapa district, where prevalence is thought to be high. ${ }^{5}$

E. granulosus tends to produce a typical large, single, round or ovoid, well-defined hydatid cyst and is the more prevalent species in sheep and cattle-raising areas, such as Central Europe, Africa, South America, New Zealand, Australia, Central Asia, and China. ${ }^{4}$ In contrast to $E$. granulosus, E. multilocularis is restricted mainly to cold and high-altitude regions, with a higher prevalence in Alaska, Canada, Central Western Europe, Siberia, and Japan. ${ }^{5}$ It causes an irregular, small, fluid-filled cavity or an invasive spongy mass resembling an infiltrating malignant hepatic tumor, rather than a well-defined expansile cyst. ${ }^{8,9}$ This patient presented with single, 
ovoid, large sized (79 X $73 \mathrm{~mm})$ hydatid cyst in the liver revealed by USG abdomen reports and hence the species E. granulosus can be considered as the causative agent.

Hydatid cyst grows $1 \mathrm{~cm}$ within first 6 months followed by $1 \mathrm{~cm}$ annually based on the host tissue resistance. ${ }^{5}$ This suggests that the patient might have got infected 3 to 4 years back. The primary cyst in the liver is composed of 3 layers namely adventia (peri cyst), laminated membrane (ectocyst) and germinal epithelium (endocyst), among which germinal epithelium if left untreated can cause recurrence. This is because of scolices present along the inside border of germinal epithelium, which are responsible for generation and development of thee other layers. ${ }^{1}$ This suggests that failure of therapeutic intervention had lead to the regeneration of cyst. Previous literature suggest that those cysts that escape the hepatic filtering can enter the lungs from where they can migrate to disseminated organs leading to several complications. ${ }^{1}$ USG chest revealing thick septations were suggestive of loculated effusion and was advised to go for CT chest to know the underlying cause. CT chest revealed that it was hydatidosis of lung with internal loculations of multiple hydatid cysts in the right lobe and a cyst with calcified wall in the left lobe, which caused pleural effusion.

Actual therapy for hydatidosis include cyst excision along with chemotherapy including ALBENDAZOLE (10 to $15 \mathrm{mg} / \mathrm{kg} /$ day in two divided doses for 3 to 6 cycles with a schedule of 4 weeks therapy and 2 weeks pause) and MEBENDAZOLE (40 to $50 \mathrm{mg} / \mathrm{kg} /$ day in 3 divided doses for 3 to 6 months) before surgery and after 8 weeks of surgery. Albendazole is an antiscolecoidal agent and highly effective for curbing the cyst growth, whereas Mebendazole is an antiheleminthic drug that has poor intestinal absorption. However, hydatid cyst of other organs requires long term therapy as available from the previous data. ${ }^{3,7}$ This patient was initially provided with Paracetamol, B.Complex, Ranitidine and augmentin as symptomatic therapy. After USG abdomen and CT Chest reports confirmed the presence of hydatid cysts in the liver and the lung, albendazole was given according to her body weight $(44 \mathrm{~kg})$ i.e. $450 \mathrm{mg}$ BD. She has been advised to continue the same therapy with review after two months. This shows that excision of the cyst along with chemotherapy before and after surgery is highly effective rather than simple excision of the cyst.

\section{CONCLUSION}

For treating the hydatid cyst, irrespective of its size, surgical therapy along with chemotherapy was proven to be highly effective from the previous studies. ${ }^{10,11}$ This case also depicts that simple surgical intervention may not be enough and requires chemotherapy to completely eliminate the cyst especially Albendazole, which acts by inactivating the scolices (live component of the cyst) and curbing the growth of cyst and thereby preventing cyst's migration to other organs. Though the exact duration of albendazole therapy for multiple organ involvement is not yet available, it depends on the size and number of cysts and also number of organs it is present in.

\section{Funding: No funding sources \\ Conflict of interest: None declared \\ Ethical approval: Not required}

\section{REFERENCES}

1. Derbel F, Ali AB, Nadia A, El Ouni C, Ibtissem H, Jemni H, et al. Hydatid cysts of the liver-diagnosis, complications and treatment. INTECH Open Access Publisher; 2012.

2. Attef $M$ Elshazly, Manar $S$ Azab, Samar N ElBeshbishi and Hany M Elsheikha. Hepatic hydatid disease - four case reports. Cases Journal. 2009;2(58);1-4.

3. Anand CL, Rajagopalan BS, Mohan SLR. Management of liver hydatid cysts e Current perspectives. Medical journal armed forces India. 2012;68:304-9.

4. Solaymani A, Jozani ZB, SeyedAlinaghi SA, Jamali SR. Multi-organ echinococcosis: a case report. Journal of International Medical Publisher. 2011;5(1):1-3.

5. Lee RC, Chou YH, Chiang JH, Chen YK and Hsu HC. Hydatid cyst of the liver: a case report and literature review. Kaohsiung Journal of Medical Sciences. 2005;21(9):418-23.

6. Faheem N, Nusrath N, Rao BSS, Ram GR, Sushma C, Subramanyam Y, et al. The scenario of Hydatid cyst disease in epidemic areas of Andhra Pradeshevaluation and analysis. International Journal of Research and development off health. 2013;(3):1208.

7. Dervenis C, Delis S, Avgerinos C, Madariaga J, Milicevic M. Changing Concepts in the Management of Liver Hydatid Disease. Joumal of Gastrointestinal Surgery. 2005;9(6):869-77.

8. Wen H, New RR, Craig PS. Diagnosis and treatment of human hydatidosis. British Journal of Clinical Pharmacology. 1993;35:565-74.

9. Biava MF, Dao A, Fortier B. Laboratory diagnosis of cystic hydatid disease. World Journal of Surgery. 2001;25:10-4.

10. Franchi C, Di Vico B, Teggi A. Long-term evaluation of patients with hydatidosis treated with benzimidazole carbamates. Journal of Clinical and Infectious Diseases. 1999;29:304-9.

11. Horton RJ. Albendazole in treatment of human cystic echinococcosis: 12 years of experience. International Journal of Infectious Diseases. Acta Trop. 1997;64:79-93.

Cite this article as: Nethi SK, Biradavolu V, Durga Prasad TS. Case report on hydatid cyst of the liver progressed to the lungs. Int J Basic Clin Pharmacol 2017;6:1003-5. 